\title{
Modelo de aplicación de AHP para seleccionar editor de contenidos de objetos de aprendizaje (modelo PAJOA - ECOA)
}

\section{RESUMEN}

EI MODELO PAJOA-ECOA.es resultado de la aplicación de conocimientos y experiencias sobre dos temas: desarrollo de objetos de aprendizaje y proceso analítico jerárquico (PAJ). La usabilidad de los objetos de aprendizaje requiere de un empaquetamiento que permita la interoperabilidad entre sistemas. Así surge la necesidad de seleccionar un editor de contenido educativo (ECE), considerando multicriterios. Una técnica adecuada para esta selección es PAJ/AHP.

Palabras clave: editor de contenido digital, PAJ, objetos de aprendizaje.

Model APPLiCATION OF AHP TO SELECT PUBLISHERS LEARNING OBJECT (MODEL PAJOA - ECOA)

\section{ABSTRACT}

The MODEL PAJOA-ECOA is the result of the application of knowledge and experencies on two topics: Development of learning objects and the analytical process hierarchy (AHP). The usability of learning objects requires a packaging that allows interoperability. Betweens systems. This way there arises the need to select a publisher of digital content considering multicriteria, a skill adapted for this selection is PAJ/AHP

Keywords: AHP, learning object, ublisher of digital content

\section{INTRODUCCIÓN}

En un marco de educación bimodal es importante brindarle al docente técnicas, herramientas para la elaboración de recursos educativos de aprendizaje (REA), en específico del desarrollo de un objeto de aprendizaje (OA). Este desarrollo de OA, implica seleccionar contenidos, y éstos deben ser transformados en contenidos digitales. Para su publicación en web se requiere una plataforma tecnológica, y a su vez para permitir la interoperabilidad enter sistemas es necesario empaquetar los contenidos, de tal modo que se puedan importar, exportar, agregar y desagregar.

Para la generación de paquetes de contenidos se tiene: el eXelearning, reload y otros, ¿cómo seleccionar el más adecuado?, ¿cuáles serían los criterios a tomar en cuenta para seleccionar el editor de contenidos educativos como los objetos de aprendizaje?

Para optimizar el proceso de selección, porque hay varios criterios a tomar en cuenta se aplica Proceso Analítico Jerárquico (PAJ)/ Analytic Hierarchy Process (AHP). Para realizar los cálculos y el análisis de sensibilidad con eficacia, existen softwares que requieren licencia como el expert choice.

\section{METODOLOGÍA}

Este trabajo es una investigación aplicada en lo concerniente a proceso analítico jerárquico (PAJ).

Por otra parte, es una investigación exploratoria sobre herramientas de contenidos educativos (ECE), específicamente en objetos de aprendizaje .

En la aplicación de MADOA [4] se ha tendio la oportunidad de utilizar eXelearning como editor de contenidos, sin embargo existen otros como Reload.

Se ha tomado en cuenta los pasos que Saaty propone sobre AHP, sin embargo ha sido necesario esquematizar, po lo cual se considera describir la metodología de aplicación PAJ/AHP, ver figura 1. Por un lado se evalúa la aplicabilidad de la técnica AHP a un caso problema, por otro lado se selecciona la herramienta de cálculo, Asimismo se describe en pasos:

* Dra. En Ingeniería. Profesora principal Universidad Nacional de Ingeniería. Dpto. Gestión de la producción. E-mail: therehuamani@gmail.com

* Mag. en Tecnología educativa. Profesor principal UNI. Profesor asociado en UNMSM. E-mail: roeyzaguirre@gmail.com 


\section{Pasos de la aplicación Metodología de aplicación PAJ/AHP:}

Paso 1: Seleccionar meta. Definido el problema es necesario tener información y experiencia de un experto sobre el tema, para seleccionar criterios y proponer alternativas.

Paso 2: Estructura jerárquica: Definido la meta global, criterios y alternativas de solución se propone el modelo Jerárquico.

\section{Paso 3. Proponer Matrices}

Matriz de criterios: La fila y columnas son los criterios. Se compara par a par. Para evitar subjetividades se utiliza la escala de comparación de Saaty. Después de haber realizado las comparaciones de todos los criterios estas matrices son normalizadas. Con esta matriz, se obtiene el vector de prioridad.

Matriz de alternativas, en esta matriz se compara las alternativas respecto a un criterio

Matriz de comparación de alternativas una vez obtenido resultados de los cálculos en las matrices de criterios y matriz de alternativas se procede a comparar las alternativas con los criterios.

Paso 4: Resultados. Se realiza el análisis de sensibilidad para evaluar la consistencia. De este modo se valida el modelo.

\section{MARCO TEÓRICO}

\subsection{Proceso de análisis jerárquico (PAJ/AHP)}

El proceso de análisis jerárquico (PAJ/AHP), es un método desarrollado por Saaty en 1977 , y propuesto de manera integrada en su libro en 1980. Saaty ha publicado diversos modelos jerárquicos aplicando a problemas complejos tanto políticos, económicos, etc. [7]

\section{Descripción de pasos AHP}

Paso 1 Desarrollo de la estructura jerárquica.

La construcción de un modelo jerárquico permite al decisor estructurar el problema en forma visual, con la técnica se busca

formalizar la comprensión intuitiva de un problema multicriterio complejo. En la Figura 2 se muestra los niveles de la jerarquía: Meta global, Criterios y alternativas.

Paso 2. Representación de los juicios de valor

Saaty [7] propone una escala de 1 a 9 , en tabla 1 , se muestra la escala verbal con su equivalente en números.

Figura 1. Metodología de aplicación PAJ/AHP.

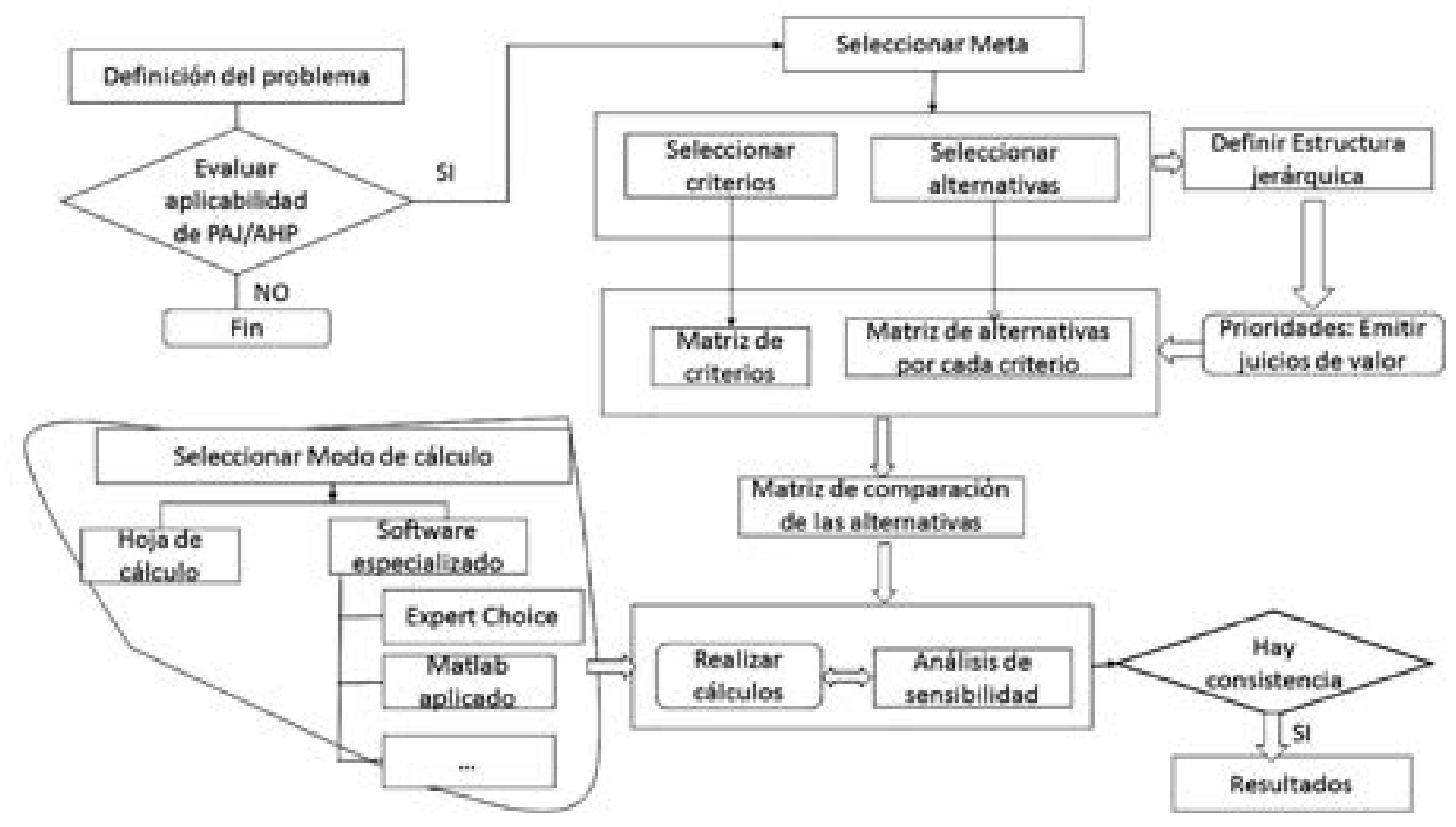


Figura 2. Modelo jerárquico.

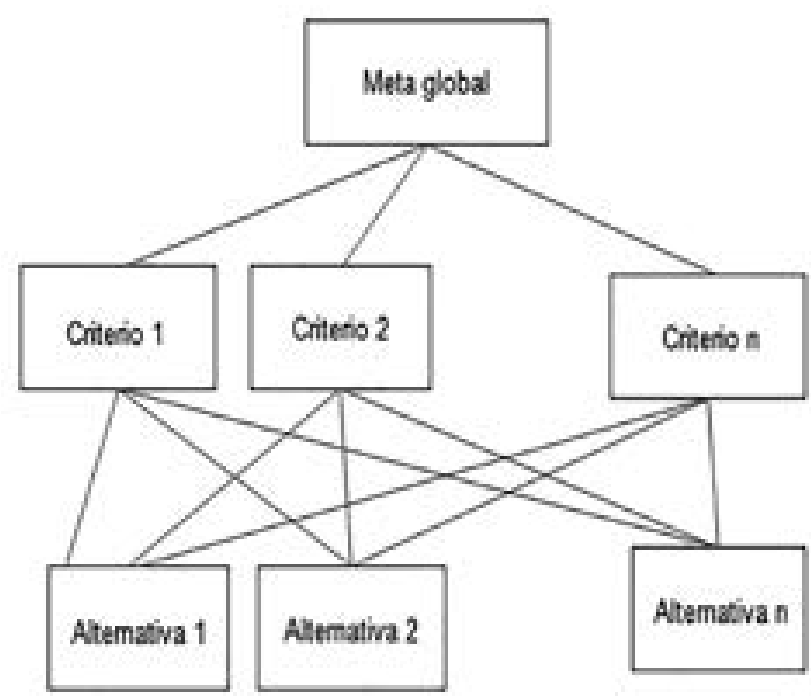

Tabla 1. Escala de Saaty

\begin{tabular}{|l|l|}
\hline \multicolumn{1}{|c|}{ Escala verbal o preferencia } & \multicolumn{1}{c|}{$\begin{array}{c}\text { Evaluación } \\
\text { numérica }\end{array}$} \\
\hline Igualmente preferido & 1 \\
\hline Moderada preferido & 3 \\
\hline Fuertemente preferido & 5 \\
\hline Muy fuertemente preferido & 7 \\
\hline En extremo preferido & 9 \\
\hline Valores intermedios & $2,4,6,8$ \\
\hline $\begin{array}{l}\text { Si a la actividad i se le ha } \\
\text { asignado uno de los números } \\
\text { distintos a cero mencionados, } \\
\text { cuando se compara con la j } \\
\text { entonces j tiene el recíproco } \\
\text { cuando se le compara con i }\end{array}$ & Recíprocos \\
\hline
\end{tabular}

(Saaty, 1980) citado por Moreno y Escobar" EI método convencional de AHP proporciona la prioridad, o importancia relativa, de los elementos comparados mediante: (a) la aplicación del método del autovector principal normalizado según el 'modo distributivo' para calcular las prioridades locales; (b) el principio de composición jerárquica para obtener las prioridades globales, y (c) la agregación multiaditiva para obtener las prioridades totales. Estas prioridades (totales) pueden ser utilizadas con distintos fines: selección de una o varias alternativas (problema tipo a); ordenación de todas ellas (problema tipo g), etc. La unicidad del vector de prioridades locales se consigue en AHP mediante la normalización" [5].

Paso 3 Construcción de las matrices de juicio de valor.

La jerarquía de criterios y alternativas es el objeto de comparación por pares para el PAJ/AHP.

Paso 4 Cálculos a desarrollar.

Una vez construida las matrices se pasa a realizar los siguientes cálculos: cálculo de los vectores y cálculo de la consistencia. A la construcción de matrices de comparaciones de pares, se le aplica el operador vector propio para derivar los pesos de los criterios, y su correspondiente valor propio, para determinar la consistencia de dichas preferencias.

Paso 5 Análisis de sensibilidad y resultados

Se calcula y se evalúa la consistencia de los criterios propuestos

\subsection{Desarrollo de objetos de aprendizaje, editores de contenidos digitales}

\subsubsection{Objetos de aprendizaje}

Hay una diversidad de definiciones, tomamos en cuenta a Chan, Galeana y Ramírez (2012) [2] Quiénes citan la definición de Wiley (2000) que un Objeto de aprendizaje es cualquier recurso digital que puede ser reusado como soporte para el aprendizaje y señalan también, "un objeto de aprendizaje es una entidad informativa digital que se corresponde (representa) con un objeto real, creada para la generación de conocimientos, habilidades, actitudes y valores, y que cobra sentido en función de las necesidades del sujeto que lo usa". .

El desarrollo de OA inicia desde la ubicación de datos, contenidos en textos, audio, animación, simulación. Si los datos son agrupados. Contextualizados, tienen un significado, entonces se convierten en objetos informativos, mostrados como procedimientos, procesos, resúmenes. Para luego convertirse en objetos de aprendizaje. Para visualizar mejor, se adaptó la taxonomía propuesta por Hodgings (2000), citado por Calzada (2010) [3] (Ver Figura 3).

Los objetos de información son pequeñas porciones de información en las que se puede dividir el conocimiento, que pueden ser combinadas y utilizadas libremente, como se hace con las piezas de LEGO. Con estos objetos es posible personalizar el aprendizaje utilizando (y reutilizando) el mismo objeto de información (Hodgings, 2000) citado por Astudillo (2011) [1] 
Figura 3. Taxonomía de objetos de aprendizaje (adaptado de Hodgings).

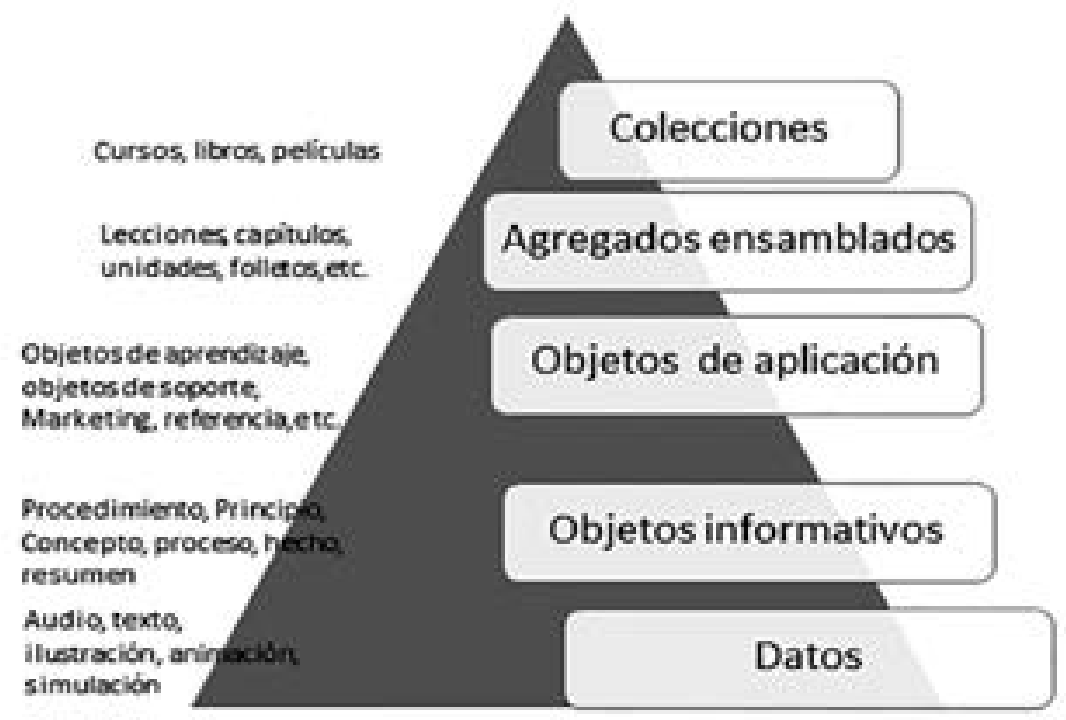

\subsubsection{Aspectos tecnológicos}

En el desarrollo de objetos de aprendizaje es necesario contar con una plataforma tecnológica para publicar el objeto de aprendizaje empaquetado y este empaquetamiento requiere de un editor de contenido educativo.

\subsubsection{Editores de Contenidos Educativos (ECE)}

Un paquete de contenidos es un archivo, generalmente comprimido en formato .zip que sigue una determinada especificación como SCORM (Sharable Content Object Reference Model) producido por ADL (Advanced Distributed Learning) o IMS LRM (Learning Resource Metadata).

Se puede elegir cualquier editor de contenido digital, están diseñados para apoyar a profesores en la producción de material educativo con formato de páginas Web. Los más conocidos son: eXelearning, Glomaker, Reload.

eXelearning. Herramienta de autor de código abierto para ayudar a los docentes en la creación y publicación de contenidos web [8]

GLO MAKER (Generative Learning Objetc Maker) [9] RELOAD (Reusable e-learning Object Authoring \& Delivery) [10]

\section{MODELO DE APLICACIÓN DE PAJ/AHP PARA SELECCIONAR EDITOR DE CONTENIDOS DE OBJETOS DE APRENDIZAJE (MODELO PAJOA -ECOA.)}

MODELO PAJOA-ECOA

1. SELECCIONAR EDITOR DE CONTENIDOS DE OBJETOS DE APRENDIZAJE

2. Desarrollo de la estructura jerárquica - Modelo jerárquico

a. Selección de criterios

Consideramos que es importante en un editor de texto la accesibilidad, disponibilidad, y facilidad de uso.

b. Alternativas de editores de texto

Para la aplicación consideramos Reload, eXelearning, y Glomaker.

c. Estructura jerárquica

Con ello proponemos el modelo jerárquico, ver Figura 4.

3. Matrices

a. Matriz calificación criterios

Criterios

Accesibilidad (C1)

Facilidad de Uso (C2)

Disponibilidad(C3)

\begin{tabular}{|c|c|c|}
\multicolumn{1}{c}{ C1 } & \multicolumn{1}{c}{ C2 } & \multicolumn{1}{c}{ C3 } \\
\hline 1 & 2 & 5 \\
\hline $1 / 2$ & 1 & 3 \\
\hline $1 / 5$ & $1 / 3$ & 1 \\
\hline
\end{tabular}

b. Matriz de alternativas respecto a un criterio

Accesibilidad

eXelearning (A1)

Reload (A2)

Glomaker(A3)

\begin{tabular}{|c|c|c|}
\hline $\mathrm{A} 1$ & $\mathrm{~A} 2$ & $\mathrm{~A} 3$ \\
\hline 1 & 5 & 6 \\
\hline $1 / 5$ & 1 & 2 \\
\hline $1 / 6$ & $1 / 2$ & 1 \\
\hline
\end{tabular}


Figura 4. Modelo PAJOA-ECOA (estructura jerárquica).

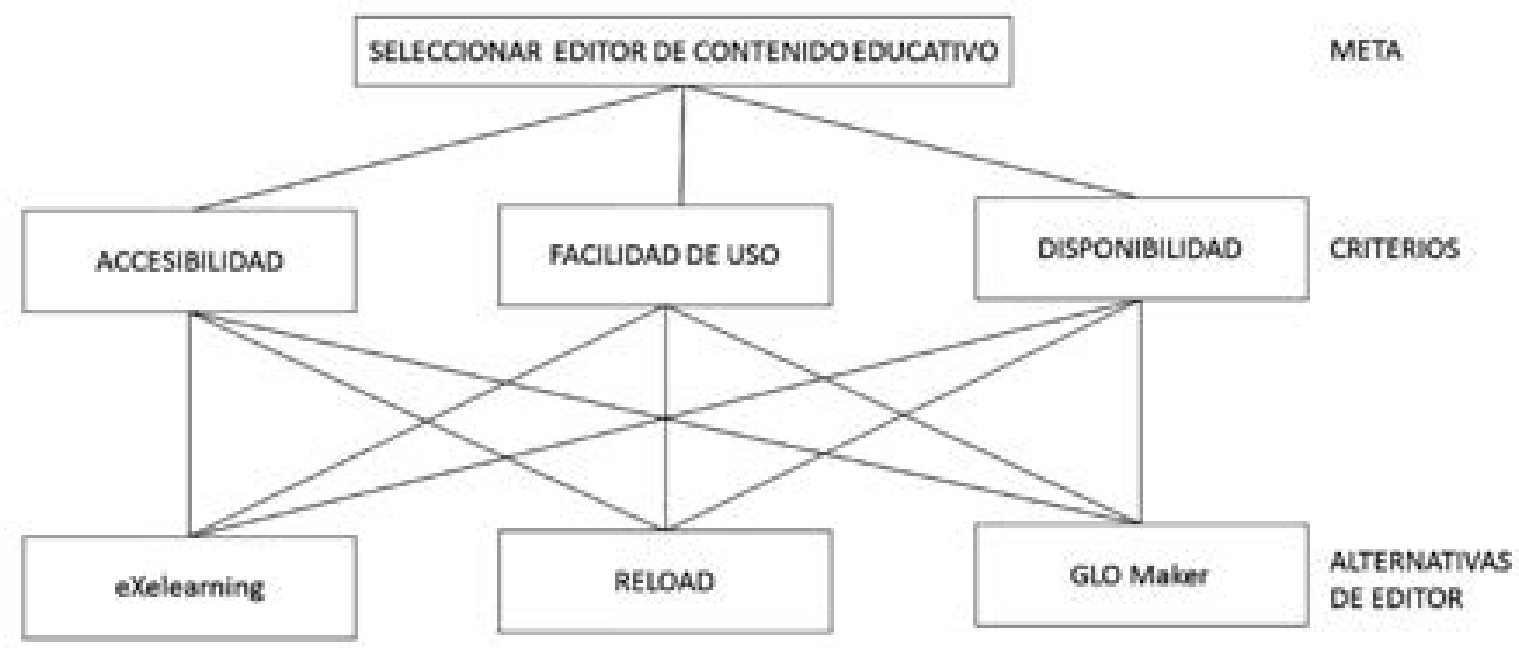

c. Matriz de comparación de alternativas

\begin{tabular}{|c|c|c|c|}
\hline & C1 & C2 & C3 \\
\hline A1 & 0.723 & 0.283 & 0.681 \\
\hline A2 & 0.174 & 0.643 & 0.118 \\
\hline A3 & 0.103 & 0.074 & 0.201 \\
\hline
\end{tabular}

\section{CONCLUSIONES}

Para formular el modelo PAJOA-ECOA se ha utilizado la metodología de aplicación PAJ/AHP, una adaptación de la formulación de Saaty. El modelo ha requerido por un lado conocimientos en desarrollo de objetos de aprendizaje, específicamente en editores de contenidos de objetos de aprendizaje. De este modo se ha propuesto criterios y alternativas de solución; por otro lado ha sido necesario contar con experiencias de uso de la técnica proceso analítico jerárquico (PAJ). La consistencia depende de los juicios de valor emitidos por lo usuarios del modelo.

\section{Agradecimientos}

Se agradece a Carlos Arréstegui, investigador sobre AHP y alumno de Logística empresarial de FIIS UNI, por haber aplicado el modelo PAJOA ECOA, utilizó la escala de Saaty para emitir juicios de valor y realizó cálculos. en Excel y en expert choice. para probar la consistencia.

\section{REFERENCIAS BIBLIOGRÁFICAS}

[1] Astudillo G.(2011). Análisis del estado del arte de los objetos de aprendizaje. Revisión de su definición y sus posibilidades. Facultad de Informática Universidad de la Plata Argentina disponible 28.01.14 http://sedici.unlp.edu.ar/ bitstream/handle/10915/4212/Documento_ completo.pdf?sequence=.

[2] Chan, ME, Galeana, L y Ramírez, MS (2012). Objetos de Aprendizaje: e innovación educativa. México. Trillas Pag.13

[3] Calzada, F. (2010). Repositorios, bibliotecas digitales y CRAl. Argentina: Alfagrama ediciones, pp. 76

[4] Huamaní, G. Modelo AHP para seleccionar proveedores de Cloud computing. TECNIA Vol 24 N. ${ }^{\circ} 2014$ Lima: Revista de la Universidad Nacional de Ingeniería, pp. 99-106

[5] Huamaní G, Eyzaguirre R y Morales M..(2015) Método Agil de desarrollo de objetos de aprendizaje para Ingeniería (MADOA). UNI FIIS: [diapositivas ]

[6] Moreno Jiménez, J. M; Escobar Urmeneta, M.T. El pesar en el proceso analítico jerárquico 1 Estudios de Economía Aplicada, vol. 14, núm. 1, abril, 2000, pp. 95-115 Asociación Internacional de Economía Aplicada Valladolid, España http:// www.redalyc.org/pdf/301/30114105.pdf

[7] Saaty,T. (1990) How to make a decisión: The Analytic Hierarchy Process" European Jourmal of Operational Research 48 North Holland. p 9-26

[8] eXelearning Disponible 07.10.14 http:// exelearning.net/

[9] GLO Maker Disponible 07.10.15 http://www. glomaker.org/.

[10]RELOAD. Disponible 07.10.15 http://www. reload.ac.uk/tools.html 